ARTICLE

\title{
Modulation of BIN2 kinase activity by HY5 controls hypocotyl elongation in the light
}

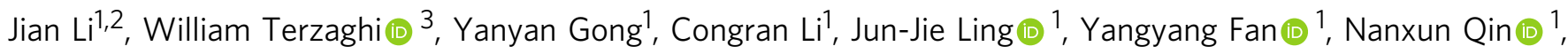
Xinqi Gong (1) ${ }^{4 凶}$, Danmeng Zhu ${ }^{1 凶}$ \& Xing Wang Deng (1,2凶

ELONGATED HYPOCOTYL 5 (HY5), a basic domain/leucine zipper (bZIP) transcription factor, acts as a master regulator of transcription to promote photomorphogenesis. At present, it's unclear whether HY5 uses additional mechanisms to inhibit hypocotyl elongation. Here, we demonstrate that HY5 enhances the activity of GSK3-like kinase BRASSINOSTEROIDINSENSITIVE 2 (BIN2), a key repressor of brassinosteroid signaling, to repress hypocotyl elongation. We show that HY5 physically interacts with and genetically acts through BIN2 to inhibit hypocotyl elongation. The interaction of HY5 with BIN2 enhances its kinase activity possibly by the promotion of BIN2 Tyr 200 autophosphorylation, and subsequently represses the accumulation of the transcription factor BRASSINAZOLE-RESISTANT 1 (BZR1). Leu ${ }^{137}$ of HY5 is found to be important for the HY5-BIN2 interaction and HY5-mediated regulation of BIN2 activity, without affecting the transcriptional activity of HY5. HY5 levels increase with light intensity, which gradually enhances BIN2 activity. Thus, our work reveals an additional way in which HY5 promotes photomorphogenesis, and provides an insight into the regulation of GSK3 activity.

\footnotetext{
${ }^{1}$ State Key Laboratory of Protein and Plant Gene Research, School of Advanced Agricultural Sciences and School of Life Sciences, Peking-Tsinghua Center for Life Sciences, Peking University, 100871 Beijing, China. ${ }^{2}$ Institute of Plant and Food Science, Department of Biology, Southern University of Science and Technology, 518055 Shenzhen, China. ${ }^{3}$ Department of Biology, Wilkes University, Wilkes-Barre, PA 18766, USA. ${ }^{4}$ Institute for Mathematical Sciences,

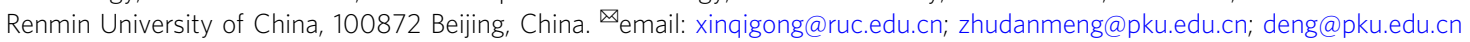


ight is not only the ultimate source of energy but also one of the most important environmental signals for plants. Light - plays a key role in the morphogenesis of Arabidopsis seedlings ${ }^{1}$. One of the most remarkable events in light-controlled morphogenesis is hypocotyl elongation. While germinating under soil without light, seedlings accelerate hypocotyl elongation in order to reach the light. Upon reaching the light, hypocotyl elongation is finely controlled to match the ambient light intensity 2,3 .

The light signal is transduced from various photoreceptors to downstream transcription factors, which regulate hypocotyl elongation by modulating transcription. HY5 is one of these lightresponsive transcription factors that play a key role in repressing seedling hypocotyl elongation ${ }^{4,5}$. It belongs to the basic leucine zipper (bZIP) family of transcription factors ${ }^{6}$. The C-terminal domain of HY5 harbors a basic region that binds DNA and a leucine zipper for dimerization ${ }^{6,7}$. HY5 regulates transcription by directly binding to $c i s$-acting elements in promoters, including the G-box, Z-box, and others ${ }^{8}$. Genome-wide ChIP-chip analyses demonstrated that HY5 directly bound the promoters of nearly 3000 genes and regulated the transcription of one-third of all genes $^{8}$. The transcriptional activity of HY5 is influenced by its physical interaction with other transcription factors. HYH, the homolog of HY5, can form heterodimers with HY5 to promote HY5 activity ${ }^{9,10}$. In contrast, the B-box-containing protein BBX28 can repress the transcriptional activity of HY5 by direct interaction $^{11}$. HY5 protein is degraded by COP1-mediated ubiquitination in the dark ${ }^{3,12}$. The COP1-HY5 module represents the core regulatory mechanism that regulates seedling morphogenesis, including hypocotyl elongation. Many other proteins, such as CALMODULIN7 (CAM7), WRKY DNA-BINDING PROTEIN 36 (WRKY36), and SHI-RELATED SEQUENCE5 (SRS5), regulate hypocotyl elongation by modulating the transcription of HY5 ${ }^{13-15}$, suggesting a central role of HY5 in the inhibition of hypocotyl elongation.

It has long been recognized that brassinosteroids (BRs) are also involved in light-controlled hypocotyl elongation ${ }^{16}$. BR-deficient mutants show shorter hypocotyls than wild type in the dark, suggesting BR promotes hypocotyl elongation ${ }^{16,17}$. As the key transcription factor responding to BR, BZR1 can promote hypocotyl elongation through its transcriptional activity ${ }^{18}$. The stability and DNA-binding activity of BZR1 protein is negatively regulated by BIN2, the Arabidopsis ortholog of human GLYCOGEN SYNTHASE KINASE 3 (GSK3) kinase. BIN2 blocks DNA binding and promotes degradation of BZR1 through phosphorylation ${ }^{19,20}$, which results in the inhibition of hypocotyl elongation.

The dwarf phenotype of bri1-5, a weak mutant of BR receptor BR INSENSITIVE 1 (BRI1), can be partially rescued by crossing it with mutants lacking PHYTOCHROME B (phyB) ${ }^{21}$, suggesting complicated regulatory interactions between light and BR signals. Several reports studying the molecular interactions involved in the cross-talk between light and BR signaling found that physical association of transcription factors acting in the two signaling pathways played a critical role in modulating hypocotyl elongation. Notably, BZR1 can directly interact with PHYTOCHROMEINTERACTING FACTOR 4 (PIF4) ${ }^{22}$. They share nearly 2000 common target genes, and act interdependently in promoting hypocotyl elongation. BZR1 can also directly regulate the level of GATA2 mRNA $^{23}$, a transcription factor repressing hypocotyl elongation acting downstream of both light and BR signals. Recent studies revealed that the photoreceptors UV RESISTANCE LOCUS 8 (UVR8) and CRYPTOCHROME 1 (CRY1) could interact with BES1 in UV-B or blue light, respectively, to inhibit its DNA-binding ability and thereby inhibit hypocotyl elongation ${ }^{24,25}$. CRY1 could also regulate BZR1 by repressing its transcriptional activity and promoting its phosphorylation by
$\mathrm{BIN}^{26}$. However, as the core factor in light signaling, the mechanism whereby HY5 regulates hypocotyl elongation in response to the cross-talk between light and BR signals remains unclear. In addition, a previous study showed that overexpression of HY5 reduced BZR1 protein levels in the light but the mechanism remains unknown ${ }^{27}$.

Here we show that in addition to its function as a transcription factor, HY5 can enhance BIN2 kinase activity through physical interaction, thereby promoting BIN2-mediated phosphorylation and degradation of BZR1, which represses BR-mediated hypocotyl elongation in the light.

\section{Results}

HY5 physically interacts with BIN2. To investigate the cross-talk between light and BR signaling, we performed yeast two-hybrid analyses and found that BIN2, the key negative regulator of BR signaling, interacted with HY5 (Fig. 1a). Likewise, the two homologs of BIN2, BIN2-Like1 (BIL1) and BIN2-Like2 (BIL2), also interacted with HY5 in yeast (Supplementary Fig. 1a). To map the subdomain of HY5 required for BIN2 interaction, we first divided the full-length HY5 CDS into $\mathrm{N}$ - and C-terminal domains and tested their interactions with His-BIN2 by in vitro pull-down assays. We found that the C-terminal domain of HY5 was sufficient for it to interact with BIN2 (Fig. 1b, c). Deletion of the leucine zipper (LZ) in the C-terminal domain of HY5 abolished the interaction between HY5 and BIN2 in vitro. Next, the in vivo interactions between full-length or truncated HY5 with BIN2 were confirmed using firefly luciferase complementation imaging (LCI) (Fig. 1d, Supplementary Fig. 1b) and coimmunoprecipitation (Co-IP) assays (Fig. 1e-g). Together, these experiments showed that the C-terminal domain of HY5, especially the LZ motif, was essential for interaction with BIN2.

HY5 genetically interacts with BIN2 and BZR1. Next, we investigated whether HY 5 could genetically interact with BIN2 by crossing hy5 into bin2-1, a gain-of-function mutant of BIN2 ${ }^{28}$. We found that the hypocotyls of double mutant hy5bin2-1 seedlings were the same length as those of bin2-1 when grown in the dark (Supplementary Fig. 2), indicating that HY5 had no effect on the short hypocotyl phenotype of bin2-1 in the dark. However, hy5bin2-1 hypocotyls were dramatically shorter than those of hy5 grown under continuous white light (cWL) (Fig. 2a). Likewise, seedlings overexpressing HY5 (HA-HY5) in bin23bil1bil2, the triple mutant of BIN2 and its two homologs, exhibited longer hypocotyls than those of HA-HY5 in cWL (Fig. 2b), suggesting that HY5 and BIN2 genetically interacted to regulate hypocotyl elongation. To further evaluate the contribution of BR signaling to the short hypocotyls of light-grown $\mathrm{HA}$ HY5 seedlings, we applied bikinin $(\mathrm{BK})^{29}$, a specific inhibitor of GSK3-like kinases including BIN2, to WT and two independent HA-HY5 lines. As shown in Fig. 2c, hypocotyls of HA-HY5 lines significantly elongated after BK treatment. In addition, the HY5 protein stability was not influenced by BIN2 in the light (Supplementary Fig. 3). Taken together, these results suggest that BIN2 and its homologs act epistatically to HY5 in the control of hypocotyl elongation.

It was previously reported that a key step in BR signaling is the phosphorylation of BZR1 by BIN2 to mark it for degradation to control hypocotyl elongation ${ }^{30}$. We therefore tested whether BZR1 was involved in the HY5-mediated inhibition of hypocotyl elongation. We used the CRISPR/Cas9 genome editing system to generate bzrlbes $1^{31}$, a double mutant knocking out both BZR1 and its homolog BES1. We then crossed bzrlbes1 into hy5 mutants, and found that the hypocotyls of the triple mutants were shorter than those of $h y 5$ (Fig. 2d). This suggested that BZR1 acts 
a

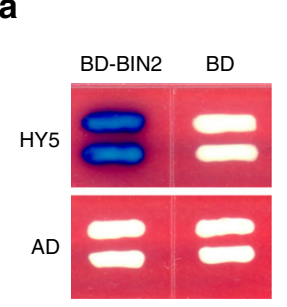

b

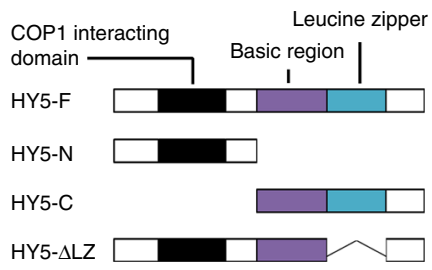

C

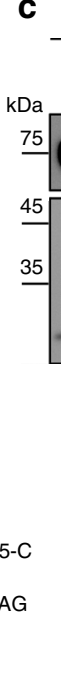

d

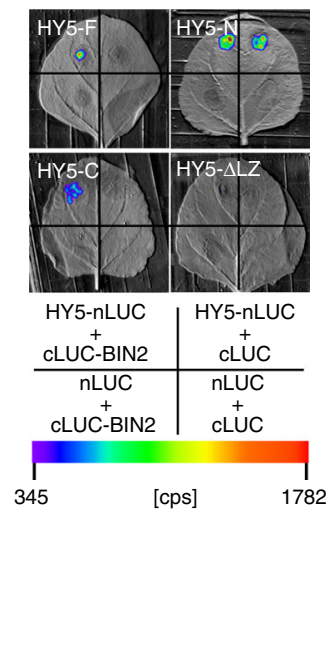

Fig. 1 HY5 interacts with BIN2. a HY5 interacts with BIN2 in yeast. Full-length HY5 was fused with AD. b Schematic representation of HY5. HY5-F, fulllength HY5; HY5-N, aa 1-77; HY5-C, aa 78-168; HY5- $\Delta$ LZ, HY5 lacking leucine zipper region (LZ, aa 115-147). c In vitro pull-down assay showing the interaction between HY5 and BIN2. Full-length and truncated HY5 were fused with the GST-tag; BIN2 was fused with the His-tag. Asterisk indicates a nonspecific band. $\mathbf{d} \mathrm{LCl}$ assay showing the interaction between $\mathrm{HY} 5$ and BIN2 in N. tabacum leaves. nLUC, the vector containing the N-terminal fragment of firefly luciferase. CLUC, the vector containing the C-terminal fragment of firefly luciferase. Full-length and truncated HY5 was fused with $\mathrm{nLUC}$ and BIN2 was fused with CLUC. The N. tabacum leaves were infiltrated with the indicated combinations. cps, counts per second. e-g Co-IP assays showing HY5 associated with BIN2. HSP was used as a loading control. Source data are provided as a source data file.

downstream of HY5 to antagonize its function in controlling hypocotyl elongation in the light.

HY5 represses BZR1 accumulation in a BIN2-dependent manner. Previous reports have suggested that HY5 and BIN2 have similar effects on BZR1 protein accumulation ${ }^{20,27}$. To gain further insight into their interplay, we checked BZR1 mRNA and protein levels in the hy5 and HA-HY5 lines. Our RT-qPCR results showed that the levels of BZR1 transcripts in hy5 and HA-HY5 were similar to those in wild type (Supplementary Fig. 4a), suggesting that HY5 did not affect the transcription of BZR1. However, the level of BZR1 protein was significantly higher in hy5 than in wild type, and this elevated level was repressed by the introduction of bin2-1 (Fig. 3a). This result suggested that HY5 might regulate the stability of BZR1 in a BIN2-dependent manner. In agreement with previous observations ${ }^{27}$, the decline in BZR1 protein level mediated by overexpression of HY5 was dependent on HY5 dosage (Supplementary Fig. 4b). Moreover, HY5 promoted the degradation of BZR1 via the 26S proteasome (Supplementary Fig. 4c). In addition, the reduced expression of BZR1 in HA-HY5 was restored when HA-HY5 was crossed into the bin2-3bil1bil2 triple mutant (Fig. 3b). Consistent with this result, the application of $\mathrm{BK}$ to the two independent $H A-H Y 5$ transgenic lines restored BZR1 protein levels to those of wild type (Fig. 3c). These results, taken together, indicate that in the light HY5 negatively regulates BZR1 accumulation in a BIN2dependent manner.

Next, to confirm whether BZR1 contributed to the altered gene expression in $h y 5$, we selected several BZR1-regulated genes involved in hypocotyl elongation and measured their expression in hy5 and hy5bzr1bes1 by quantitative real-time PCR (qPCR). Our results showed that the altered expression of these genes in hy 5 could be partially restored by the absence of BZR1 and BES1 (Fig. 3d). This indicated that part of the way in which HY5 regulates expression of genes controlling hypocotyl elongation is by destabilizing BZR1.
HY5 interaction with BIN2 enhances BIN2 kinase activity. To further identify the mechanism by which HY5 regulated BIN2 in the light to destabilize BZR1, we first checked whether HY5 regulated the mRNA and/or protein levels of BIN2. As shown in Supplementary Fig. 5, both the mRNA and protein levels of BIN2 were similar in hy5 and WT, suggesting that HY5 does not affect the expression of BIN2. Next we tested whether HY5 influenced the interaction of BIN2 with BZR1 by in vivo Co-IP assays. These showed that the amounts of BZR1 Co-IPed by BIN2-FLAG were similar in hy5 and wild type (Supplementary Fig. 6), indicating that HY5 had no effect on the interaction between BIN2 and BZR1. This prompted us to test whether HY5 modulated the kinase activity of BIN2 to regulate the phosphorylation of BZR1. We therefore performed in vitro kinase assays with His-BIN2 and MBP-BZR1, and found that BIN2 directly phosphorylated BZR1 in our system (Fig. 4a). Interestingly, we found that the phosphorylation levels of BZR1 were positively correlated with the amounts of HY5-His added to the kinase reactions (Fig. $4 \mathrm{a}$, Supplementary Fig. 7), suggesting a synergistic effect of HY5 on BZR1 phosphorylation by BIN2. In addition, the autophosphorylation level of BIN2 was also significantly increased (Fig. 4a), suggesting that HY5 upregulated BIN2 activity in a dosage-dependent manner. To confirm this effect of HY5 in vivo, we performed cell-free kinase assays using total lysates of wild type and hy5 seedlings supplemented with equal amounts of HisBIN2 and MBP-BZR1. The phosphorylation levels of BZR1 were clearly higher in WT than in hy5 extracts prepared from lightgrown seedlings but not in the extracts prepared from the seedlings transferred from light to the dark (Fig. 4b). This suggests that the phosphorylation of BZR1 by BIN2 is enhanced in the presence of HY5 in the light.

To further confirm that the enhancement of BIN2-mediated BZR1 phosphorylation by HY5 is dependent on HY5-BIN2 interactions, we conducted in vitro kinase assays using derivatives of truncated recombinant HY5 proteins. Our data showed that adding either the full-length HY5 (HY5-F) or C-terminal domain of HY5 (HY5-C) could increase BIN2-mediated phosphorylation 
a
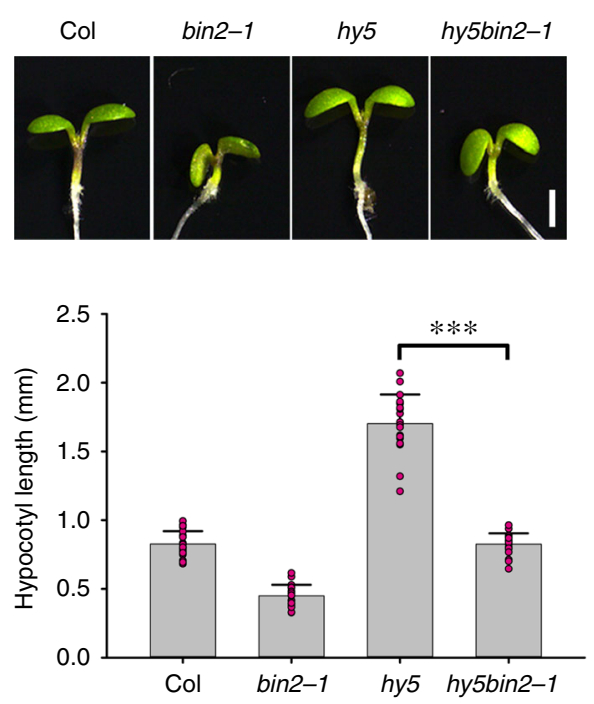

C
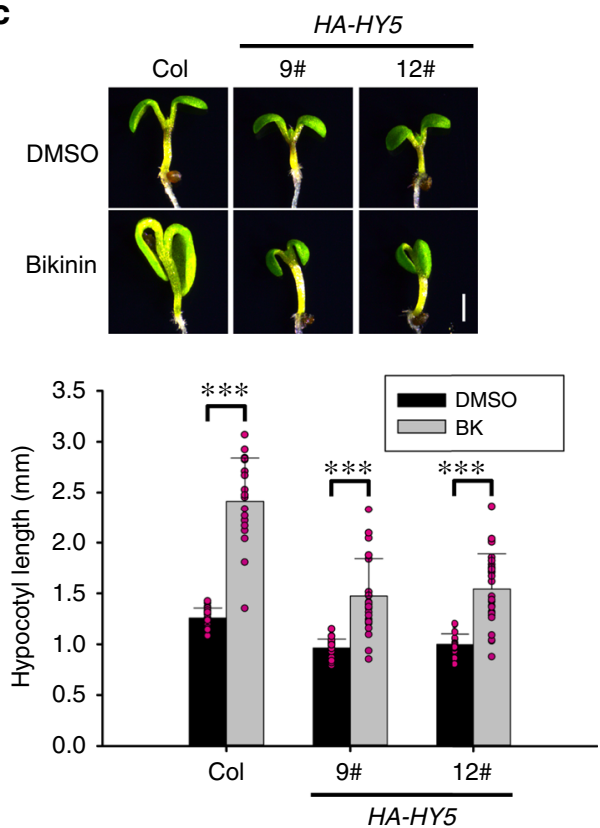

b
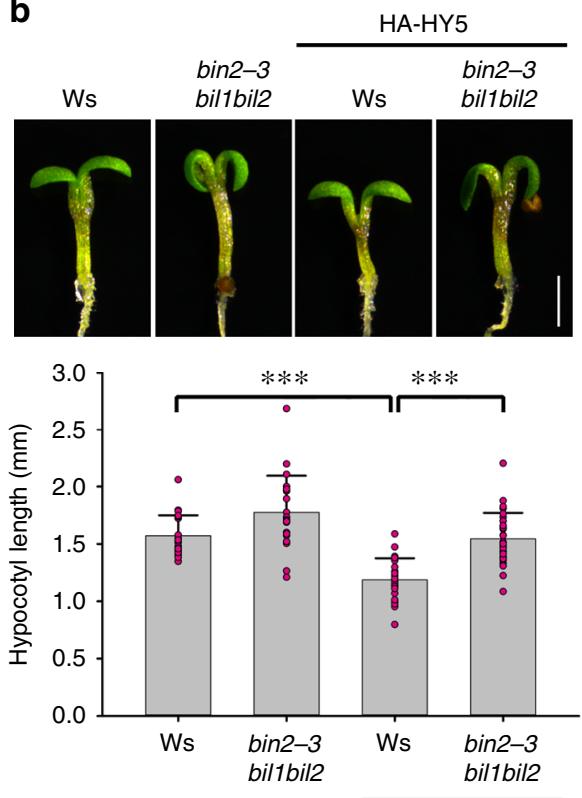

HA-HY5

d
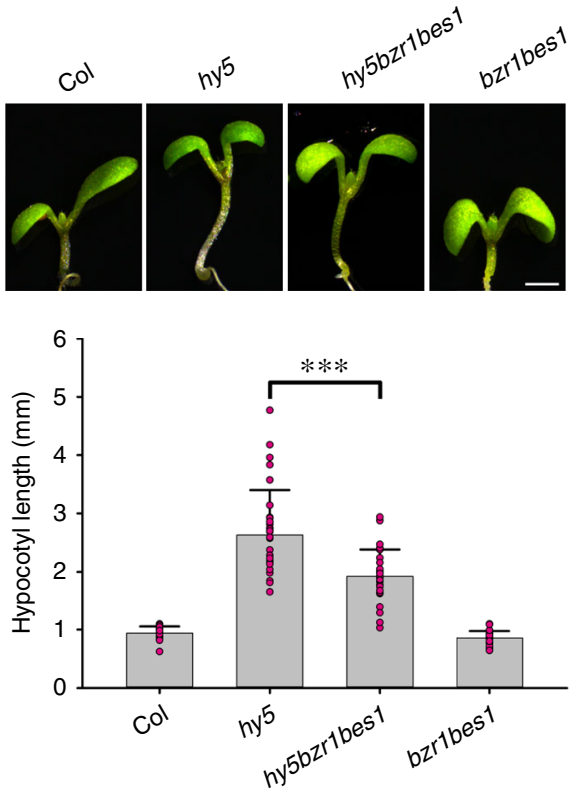

Fig. 2 HY5 genetically interacts with BIN2 and BZR1 to inhibit hypocotyl elongation in the light. a Hypocotyl lengths of Col, bin2-1, hy5, and hy5bin2-1 mutant seedlings. b Hypocotyl lengths of lines overexpressing HY5 in Ws and bin2-3billbil2 backgrounds. bin2-3bil1bil2, null mutant of BIN2 and its closest homologs, BIL1 and BIL2. HA-HY5, transgenic seedlings overexpressing $3 \times H A$-tagged HY5. c Hypocotyl lengths of HA-HY5 line seedlings treated with $30 \mu \mathrm{M}$ bikinin (BK), an inhibitor of Arabidopsis GSK3-like kinases. d Hypocotyl lengths of light-grown seedlings of hy5bzr1bes1 and bzr1bes1. BZR1 and BES1 double mutants were generated by CRISPR/Cas9. Seedlings were grown for 6 days under continuous white light (cWL). Seedlings were grown under $20 \mathrm{~W}$ m ${ }^{-2}$ (a, d) or $10 \mathrm{~W} \mathrm{~m}^{-2}(\mathbf{b}, \mathbf{c})$ light intensity, respectively. Error bars represent standard deviation (SD), $n \geq 20$. Asterisks indicate the $P$ value of two-tailed Student's $t$-test; ${ }^{\star \star \star} P<0.001$. Experiments were performed three times with similar results. Bar $=1 \mathrm{~mm}$. Source data are provided as a source data file.

levels of BZR1 to about two-fold higher than the control (Fig. 4c). Furthermore, inhibition of HY5-BIN2 binding by expressing HY5 lacking the LZ region completely abolished its capacity to upregulate BIN2 activity (Fig. 4c). This emphasizes the importance of HY5-BIN2 interactions in HY5 modulation of BIN2 activity. Next, we generated transgenic plants overexpressing either HY5-F or HY5-C in the hy5 mutant background. As expected, overexpression of HY5-C or HY5-F in the hy5 background could suppress the over-accumulation of BZR1 in hy5, while overexpression of HY5- $\Delta \mathrm{LZ}$ in hy5 failed to inhibit the over-accumulation of BZR1 (Fig. 4d). These results support the notion that binding of HY5 via its LZ region to BIN2 is essential for enhancing BIN2 activity. This increases the phosphorylation and degradation of BZR1 in the light.

HY5 L137D mutation reduces BIN2 activity. To understand which amino acid residues of HY5 were important for its interaction with BIN2, we screened BIN2-interacting sites in the HY5 LZ region. As shown in Fig. 5a, changing HY5 Leu ${ }^{137}$ (L137) to Asp (named HY5-M) attenuated interaction of HY5 with BIN2 in 

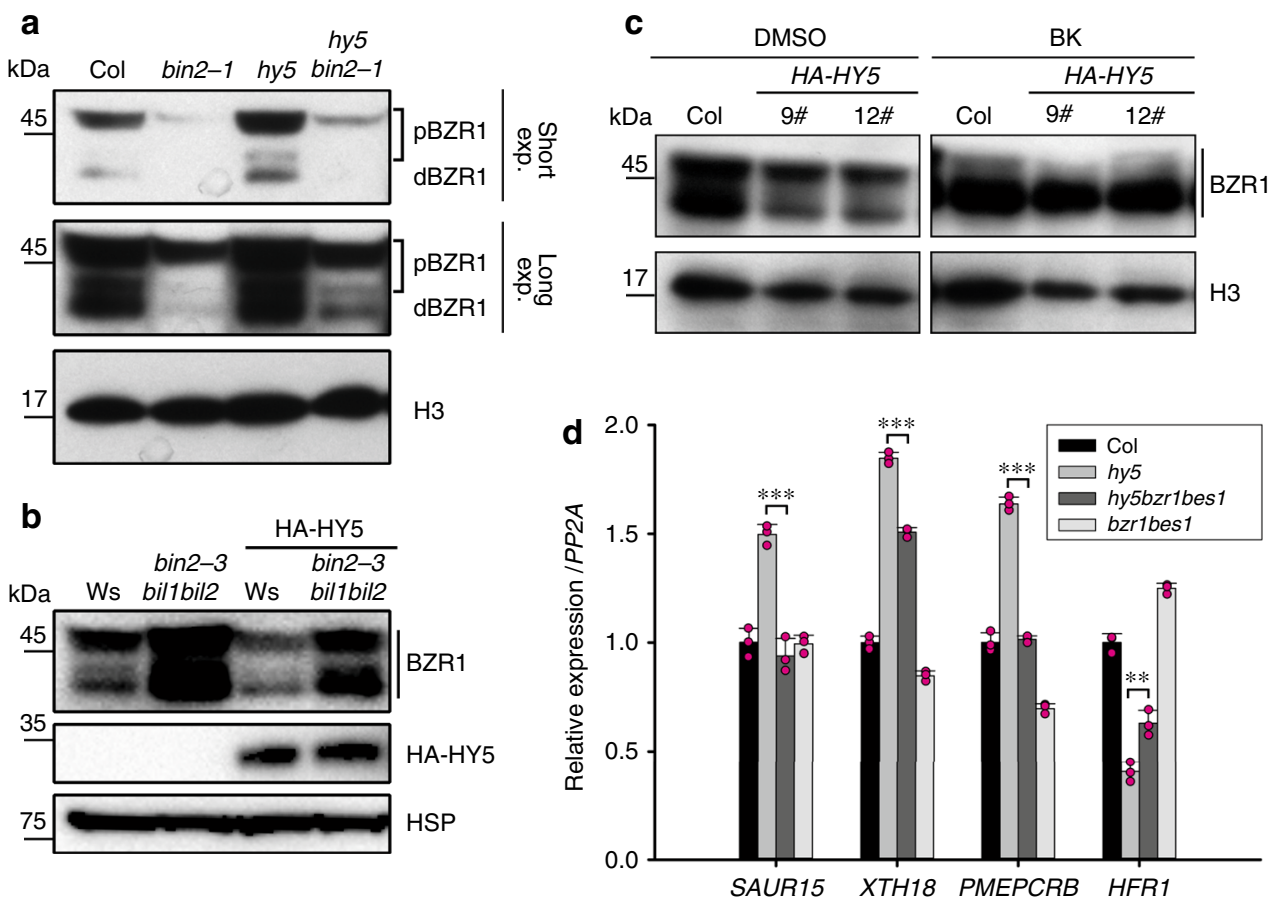

Fig. 3 HY5 represses the accumulation of BZR1 in a BIN2-dependent manner in the light. a Protein levels and phosphorylation status of BZR1 in Col and the indicated mutants. bin2-1, gain-of-function mutant of BIN2. Short exp., short exposure. Long exp., long exposure. pBZR1, the phosphorylated form of BZR1; dBZR1, the dephosphorylated form of BZR1. H3 was used as a loading control. b BZR1 protein levels in HA-HY5/Ws and HA-HY5/bin2-3billbil2 respectively. HSP was used as a loading control. c BZR1 protein levels in HA-HY5 lines treated with $10 \mu \mathrm{M} B K$. H3 was used as a loading control. d Relative expression of genes regulated by BZR1 in Col and the indicated mutants. The relative expression levels were normalized to PP2A. Error bars represent SD, $n=3$. Asterisks indicate the $P$ value of two-tailed Student's $t$-test; ${ }^{\star \star} P<0.01 ;{ }^{\star \star \star} P<0.001$. RT-qPCR was performed three times with similar results. Source data are provided as a source data file.

yeast. In vitro pull-down and LCI assays confirmed the attenuated interaction between HY5-M and BIN2 (Fig. 5b, c). Together with these results, we demonstrated that the L137 site of HY5 is important for its interaction with BIN2.

Next, we chose the promoter of EXP2, one of the target genes of $\mathrm{HY}^{32}$, to test transcriptional activation activity of HY5-M. Yeast one-hybrid assays showed that HY5-M protein could bind to the EXP2 $2_{\text {pro }}$ as well as HY5-WT (Fig. 5d). Protoplast transient expression assays in Arabidopsis showed that HY5-M could activate the transcription of EXP $2_{\text {pro }}$ : $L U C$ at normal levels (Fig. 5e, f). Taken together, L137 of HY5 is not necessary for its transcriptional activation activity.

Because of the attenuated interaction between HY5-M and BIN2, we performed in vitro kinase assays to test whether HY5-M could regulate the kinase activity of BIN2. However, adding HY5$M$ protein did not obviously increase the phosphorylation level of BZR1 (Fig. 5g), indicating that HY5-M greatly reduced the ability to regulate BIN2 activity in vitro. Consistent with this, the dephosphorylated forms of BZR1 accumulated to higher levels in HY5-M/hy5 lines than in HY5-WT/hy5 (Fig. 5h). Likewise, the hypocotyls of HY5-M/hy5 lines were significantly longer than those of HY5-WT/hy5 (Fig. 5i).

HY5 facilitates the autophosphorylation of BIN2 Y200. To gain further insights into the mechanism by which HY5 modulates BIN2 activity, we analyzed the structure of the HY5-BIN2 complex by the computational protein-protein docking method. We found that HY5 interacts with BIN2 through hydrophobic interactions between L137 of HY5 and V247 and F277 of BIN2 (Fig. 6a), in agreement with our finding that L137 of HY5 was important for the HY5-BIN2 interaction.
When we further studied the structure of the HY5-BIN2 complex by coarse-grained functional simulations we found that the amplitude of the functional motion between the $\mathrm{N}$ and $\mathrm{C}$ domains of BIN2 was much greater in the presence of HY5 (Fig. 6b, Supplementary Movies 1 and 2). It is worth noting that with HY5, the direction of BIN2 motion also changed from twist to open-close, which lead to the intramolecular approach of active catalytic sites of BIN2 to its Y200 residue. Given that the intramolecular phosphorylation of BIN2 $\mathrm{Y}^{200}$ residue $\left(\mathrm{pTyr}^{200}\right)$ is critical for its kinase activity ${ }^{33}$ and that the access of Y200 to active catalytic pocket should promote the autophosphorylation of Y200 of BIN2 (Fig. 6b), we speculated that HY5 may affect the level of $\mathrm{pTyr}^{200}$ in vivo. Indeed, when we used a specific antibody for detecting $\mathrm{pTyr}^{200}$ of BIN2 $2^{33}$, we found that the lack of HY5 led to a decline in pTyr $^{200}$ level, suggesting that the HY5-BIN2 interaction was important for BIN2 activity.

Light adjusts HY5-mediated regulation of BIN2 activity. It has been shown in Arabidopsis that increased light intensities result in the gradual accumulation of HY5 proportional to the reduction in hypocotyl length ${ }^{3}$ (Fig. 7a). We therefore determined the effect of varying light intensities on BZR1 phosphorylation and stability regulated by the HY5-BIN2 module. The levels of BIN2-F protein were very similar in the seedlings grown under increasing light intensities (Fig. 7b). We then analyzed the abundance of BZR1 in WT and hy 5 mutant seedlings. Interestingly, as the light intensity increased, the levels of BZR1 protein decreased in WT. The rate of this decrease was obviously slower in hy5, suggesting that light intensity regulated BZR1 stability in a partially HY5-dependent manner (Fig. 7c). In agreement with this result, using cell-free kinase assays we found that the BZR1 phosphorylation levels were much higher in WT lysates than in those of hy5 extracted from 
a

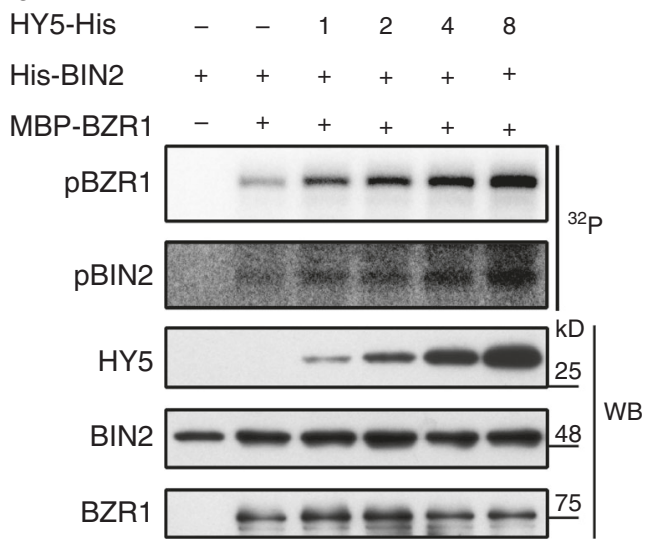

C

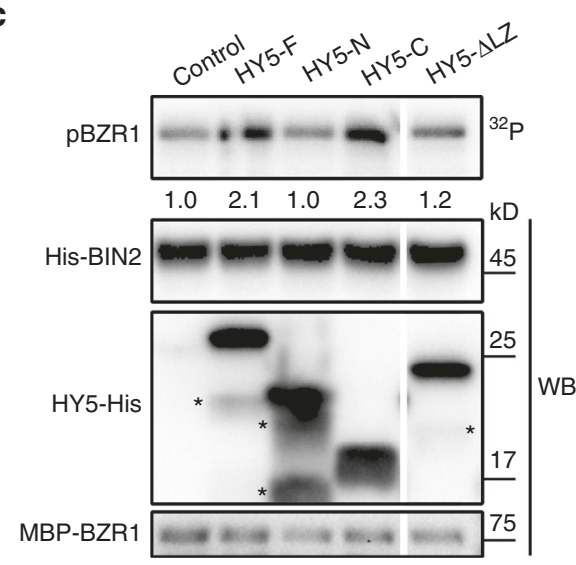

b

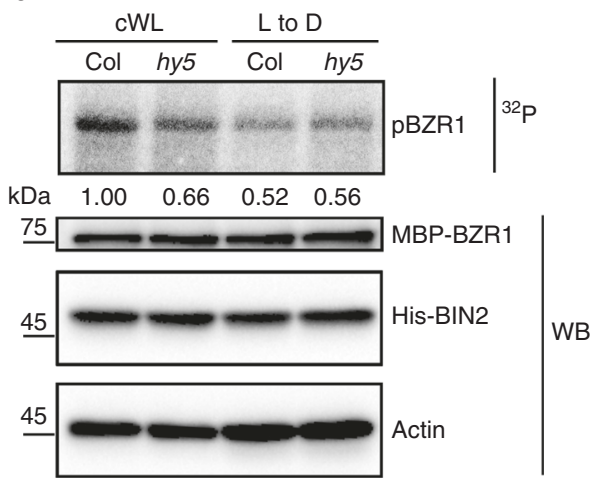

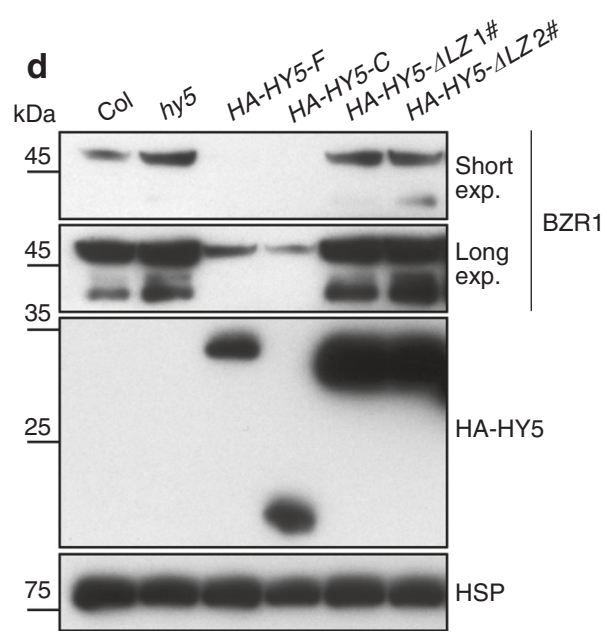

Fig. 4 HY5 enhances BIN2-mediated phosphorylation of BZR1 through its C-terminal domain. a In vitro kinase assay showing HY5 enhanced kinase activity of BIN2. MBP-BZR1 was used as substrate for BIN2. 1, 2, 4, 8 indicate the mole ratios of HY5-His versus His-BIN2. pBZR1, phosphorylated BZR1; pBIN2, auto-phosphorylated BIN2. ${ }^{32} \mathrm{P}$, autoradiography of $\left[\gamma^{-32} \mathrm{P}\right]$ ATP-labeled proteins. WB, western blot of proteins used in the kinase assay. $\mathbf{b}$ Cell-free kinase assay showing BIN2-mediated phosphorylation of BZR1 in the total lysates of Col and hy5. Seedlings grown in cWL were grown for another day in $\mathrm{cWL}$, or transferred to the dark ( $L$ to $D$ ) for 1 day. Actin was used as a loading control. c Mapping the region of HY5 that was essential for the promotion of BIN2 activity in vitro. Asterisks indicate non-specific bands. d BZR1 protein levels in transgenic plants that overexpressed full-length and truncated HY5 in the hy5 background. Short exp., short exposure. Long exp., long exposure. HSP was used as a loading control. Numbers under lanes in $\mathbf{b}$ and $\mathbf{c}$ indicate relative band intensities that were quantified for each panel. Source data are provided as a source data file.

seedlings grown under increasing light intensities (Fig. 7d). These results demonstrated that light intensity promoted BIN2mediated BZR1 phosphorylation and degradation at least in part by controlling HY5 abundance.

To further determine the effect of the HY5-BIN2 module on regulating hypocotyl elongation, we compared the hypocotyl lengths of bin2-1, hy5, and hy5bin2-1 seedlings grown under various light intensities. The hypocotyl lengths of bin2-1 and hy5bin2-1 were comparable in the dark (Fig. 7e). Under low light $\left(1.7 \mathrm{~W} \mathrm{~m}^{-2}\right)$, the hypocotyls of hy5bin2-1 were slightly longer than bin2-1, but still much shorter than wild type. In contrast, under high light $\left(20 \mathrm{~W} \mathrm{~m}^{-2}\right)$, the hypocotyls of hy5bin2-1 were much longer than bin2-1, and were as long as wild type (Fig. 7e). Taken together, HY5 increasingly contributed to BIN2 activity as light intensity increased. Therefore, by responding to varying light intensity, seedlings could adjust hypocotyl elongation by modulating the HY5-mediated regulation of BIN2 activity.

\section{Discussion}

To date, HY5 has been shown to function as a transcription factor that regulates gene expression. Many genes involved in hypocotyl elongation are the targets of HY5, such as LONG HYPOCOTYL IN FAR-RED 1 (HFR 1$)^{8}$, FAR-RED ELONGATED HYPOCOTYL 1
$(\mathrm{FHY} 1)^{34}$, INDOLE-3-ACETIC ACID INDUCIBLE 19 (IAA19) $^{35}$, EXPANSIN 2 (EXP2) $)^{35}$. There is no doubt that HY5 is a high level regulator of the transcriptional cascades controlling photomorphogenesis ${ }^{8}$. However, in this study, we revealed that HY5 repressed BR-mediated hypocotyl elongation in a way distinct from its function as a transcription factor. At low light intensities, the abundance of HY5 is limited by COP1-mediated ubiquitination and degradation. BIN2 was at least in part inactive at low HY5 levels, which led to the accumulation of BZR1 and its transcriptional activation of genes promoting hypocotyl elongation. As the light intensity increased, HY5 could accumulate due to the inactivation of COP1, thereby enhancing BIN2 activity through physical interaction. This sequentially promoted BIN2-mediated phosphorylation and degradation of BZR1 to inhibit transcription of genes downstream of BZR1, with the end result of repressing Arabidopsis hypocotyl elongation. Moreover, with increasing light intensity, an increasing amount of HY5 protein could bind BIN2 to greater enhance its activity (Supplementary Fig. 8). Therefore, in order to respond to varying light intensities, plants have used the flexible HY5-BIN2-BZR1 cascade to subtly adjust hypocotyl growth.

It has long been uncertain whether light regulates BR signaling or BR regulates light signaling. Restricted by insufficient energy 
a

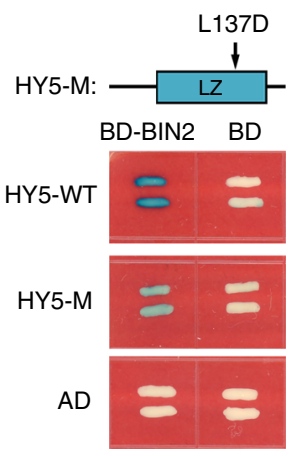

b

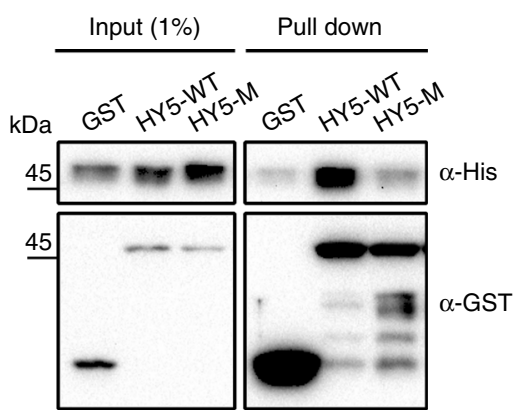

d

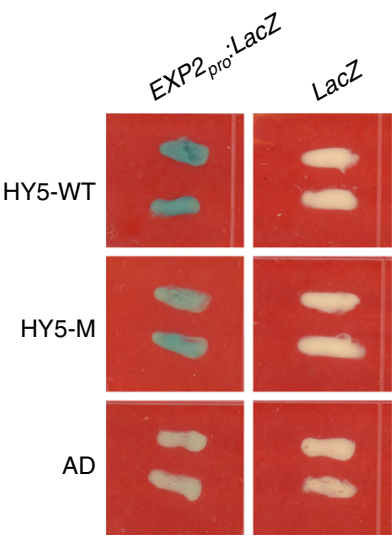

e

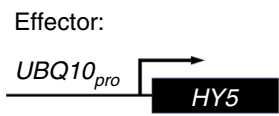

Reporter:

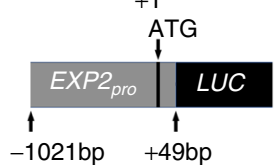

Internal control:

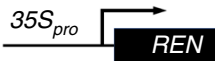

C

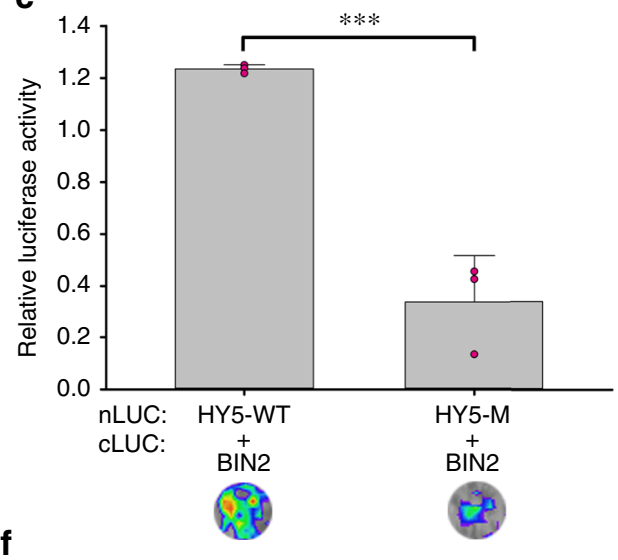

g

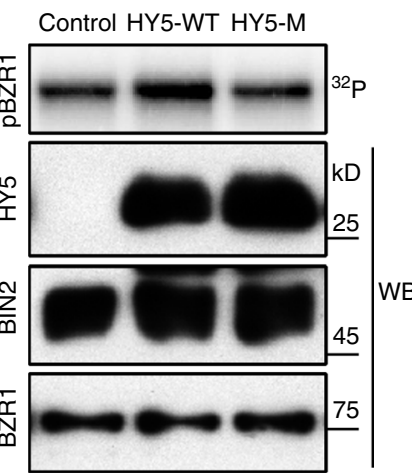

h

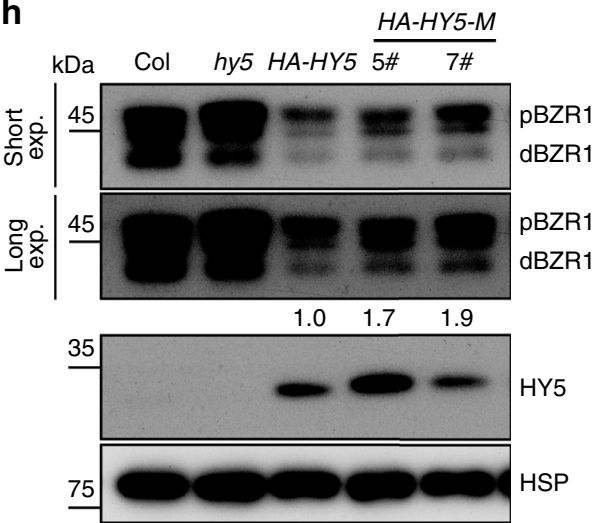

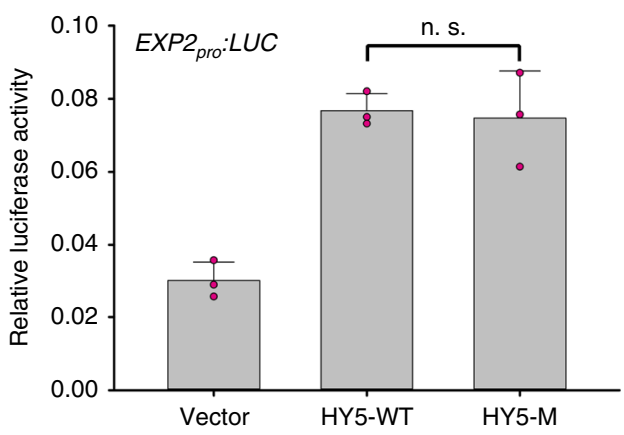

i

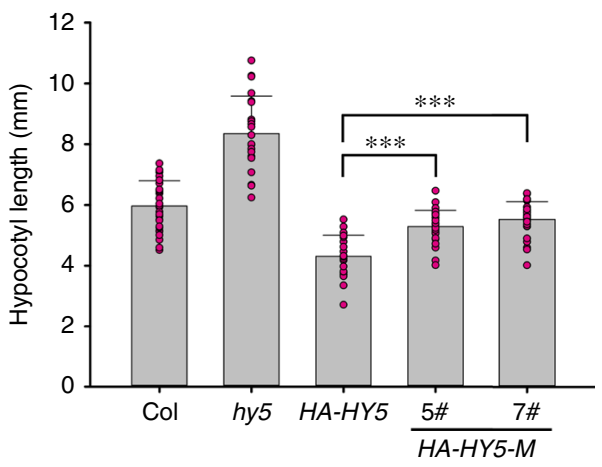

Fig. 5 HY5 can regulate the kinase activity of BIN2 independently of its transcriptional activity. a The attenuated interaction between BIN2 and mutated HY5 (HY5-M) in yeast. WT wild type. b In vitro pull-down assay showing attenuated interaction between BIN2 and $\mathrm{HY} 5-\mathrm{M}$. c LCl assay showing attenuated interaction between BIN2 and HY5-M in N. tabacum leaves. The values measuring the interaction between HY5 and BIN2 were obtained by subtracting the interaction between HY5 and the cLUC empty vector. Error bar represents SD, $n=3$. Asterisks indicate the $P$ value of two-tailed Student's $t$-test; ${ }^{\star \star \star} P<0.001$. Experiments were performed three times with similar results. d Yeast one-hybrid assay showing HY5-M could bind to the EXP2 promoter in yeast similarly to HY5-WT. HY5 was fused with the activation domain (AD). The EXP2 promoter was inserted upstream of the LacZ reporter gene. e Schematic representation of various constructs used in the transient transfection assay in Arabidopsis protoplasts. LUC, firefly luciferase. REN, renilla luciferase. f Relative LUC expression level driven by EXP2 $2_{\text {pro }}$ in Arabidopsis protoplasts. The gene expression levels were quantified as the ratios of LUC/REN enzyme activities. Error bar represents SD of three independent transient transfections in protoplasts. n.s., not significant in two-tailed Student's t-test. Experiments were performed three times with similar results. $\mathbf{g}$ In vitro kinase assay showing HY5-M could not enhance BIN2 activity. Control, no recombinant HY5 was added to the reaction. $\mathbf{h}$ The abundance of the dephosphorylated form of BZR1 in HA-HY5-M lines. Numbers below the bands indicated the relative band intensities of dBZR1. pBZR1, phosphorylation form of BZR1; dBZR1, dephosphorylation form of BZR1. HA-HY5 or HA-HY5-M were overexpressed in the hy5 mutant background. HSP was used as a loading control. i Hypocotyl lengths of HA-HY5/hy5 and HA-HY5-M/hy5, respectively, in the light. Seedlings were grown for 6 days in the light. Error bars represent SD, $n \geq 20$. Asterisks indicate the $P$ value of two-tailed Student's $t$-test; ${ }^{\star \star \star} P<$ 0.001. Experiments were performed three times with similar results. Source data are provided as a source data file. 

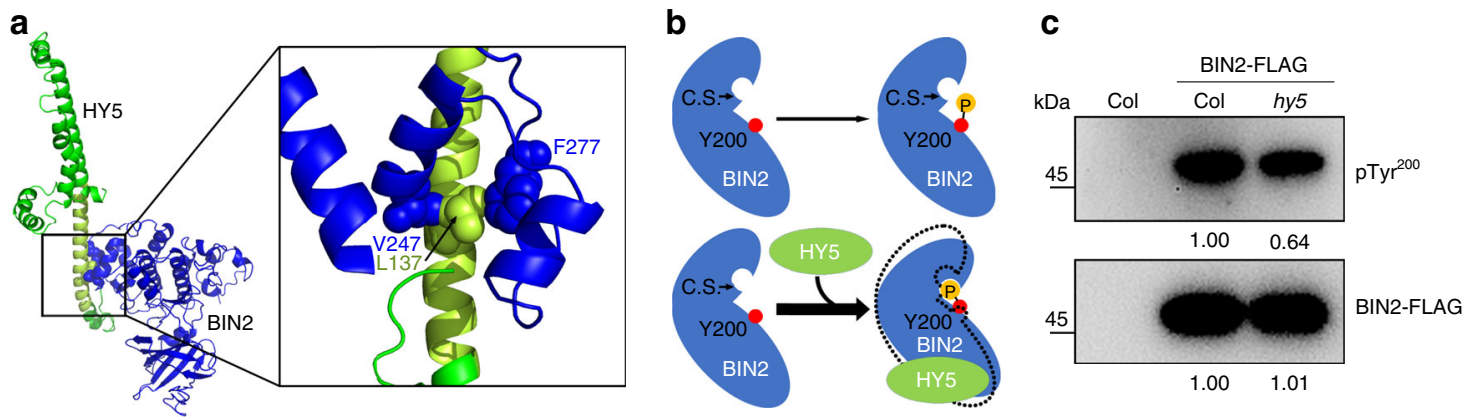

Fig. 6 HY5 promotes the autophosphorylation of the BIN2 Y200 residue. a (Left) The interaction of HY5 (green) with BIN2 (blue) predicted by computational protein-protein docking. HY5 LZ region, pale green. (Right) Zoom in on the interaction between HY5 and BIN2. Blue sphere, V247 and F277 of BIN2 respectively. Pale green sphere, L137 of HY5. b A cartoon diagram showing the intramolecular movement of BIN2 in the presence of HY5. C.S., active catalytic sites. $\mathrm{P}$ in yellow ball, phosphate group. Y200 residue of BIN2 is shown as red ball. The dotted lines represent the structural state of BIN2 before binding with HY5. The thickness of long arrows indicates the relative autophosphorylation level of Y200 of BIN2. c Immunoblots showing the levels of BIN2 pTyr 200 in vivo. BIN2-FLAG immunoprecipitated by anti-FLAG antibody were used to detect the pTyr ${ }^{200}$ of BIN2. Numbers under each lane indicate the band intensities of $\mathrm{pTyr}^{200}$ or total BIN2-FLAG, respectively. Source data are provided as a source data file.

and resources, plants must balance their growth with survival. Therefore, hypocotyl elongation must be precisely regulated, since the coordination of light signaling with signaling by internal hormones (including BR) is important for plant survival and growth. Previous work showed that addition of BL did not affect HY5 stability ${ }^{27}$. In turn, our work showed that HY5 destabilized BZR1 by promoting the kinase activity of BIN2 in the light, indicating BR signaling acted epistatically to light signaling. Recent studies revealed that the light-repressed transcription factors PIF4 and PIF5 bound to the promoter regions of key BR biosynthetic genes to directly promote their expression, thereby inducing BR synthesis ${ }^{36,37}$. Taken together, light signaling may repress BR signaling by inhibiting PIF-induced BR synthesis and promoting HY5-induced BIN2 activity in Arabidopsis.

Previously, several key players have been identified in the regulation of BIN2 stability or activity. For instance, BRI1 SUPPRESSOR 1 (BSU1) and HISTONE DEACETYLASE 6 (HDA6) repress BIN2 activity through dephosphorylation and deacetylation of BIN2, respectively ${ }^{33,38}$, while OCTOPUS (OPS) directly represses BIN2 function by sequestering it to the plasma membrane $^{39}$. KINK SUPPRESSED IN bzr1-1D (KIB1) acts as an Fbox E3 ubiquitin ligase that promotes the degradation of BIN $2^{40}$. However, little is known about positive regulators promoting BIN2 activity. Our work identified HY5 as one of the positive regulators important for the regulation of BIN2 activity in vivo. It is interesting that we observed the strong effect of HY5 overexpression on the reduction of BZR1 expression while the upregulation of BZR1 in the hy5 mutant is relatively mild (Figs. 3 and 4). These data suggest that HY5 may have functional homologs in modulating BIN2-dependent regulation of BZR1. It will be of great interest to define and characterize these factors in the future study.

Previous studies have shown that Y216 in GSK3 $\beta$ is conserved in all GSK3s identified so far, and its phosphorylation is essential for full kinase activity of GSK3s $s^{41,42}$. Y216 phosphorylation in GSK3 $\beta$ was an intramolecular autophosphorylation event in mammalian cells ${ }^{43}$, and required the presence of HEAT SHOCK PROTEIN 90 (HSP90) ${ }^{44}$. In this study, we found that, similar to HSP90 in humans, HY5 plays a role in promoting the autophosphorylation of BIN2 Y200, the homolog of GSK3 $\beta$ Y216, to modulate its kinase activity. Our work expands the understanding of regulation of GSK3 kinase activity in general.

\section{Methods}

Plant materials and growth conditions. The ecotypes of all wild-type Arabidopsis thaliana used in this study were Columbia-0 (Col) and Wassilewskija-2 (Ws). The bin2-1, bzr1bes1, hy5, and bin2-3bil1bil2 mutants were reported previously $17,31,45,46$. Seeds were sterilized with $15 \%$ bleach. After 2 days of stratification at $4{ }^{\circ} \mathrm{C}$, seeds were grown on MS medium ( $\mathrm{pH}$ 5.7) supplemented with $1 \%$ sucrose and 0.6\% Agar (A1296; Sigma-Aldrich). Six-day-old seedlings grown under cWL were used in this study unless otherwise indicated. For MG132 or BK treatment, seedlings grown in the light were transferred to liquid MS solution supplemented with $30 \mu \mathrm{M}$ MG132 or $10 \mu \mathrm{M}$ BK, respectively, for $6 \mathrm{~h}$ before harvest.

Plasmid construction and generation of transgenic lines. To generate the pLacZi-EXP2 $2_{\text {pro }}$ construct for yeast one-hybrid assays, the subfragment of the EXP2 promoter $^{32}$ was amplified and inserted into the KpnI/XhoI sites of the pLacZi vector ${ }^{47}$.

To generate pB42AD-HY5 constructs for yeast two-hybrid assays, fragments containing full-length $H Y 5$ coding sequence (CDS) (1-168 aa), and truncated HY5 CDS including HY5-N (1-77 aa), HY5-C (78-168 aa), and HY5- $L L Z$ (deletion of $115-147 \mathrm{aa})$ were amplified and inserted into the EcoRI/XhoI sites of the pB42AD vector (Clontech). For the BD-BIN2, BD-BIL1 and BD-BIL2 constructs, DNA fragments encoding full-length $B I N 2, B I L 1$, and BIL2 CDS were amplified and inserted into the EcoRI/Bam HI sites of the pLexA vector (Clontech).

For purification of His-BIN2, GST-HY5, and HY5-His recombinant proteins, the full-length BIN2 CDS fragment was inserted into the EcoRI/SalI sites of the pET28a vector, full-length and truncated HY5 fragments were inserted into the EcoRI/XhoI sites of the pGEX4T-1 vector or NcoI/NotI sites of the pET28a vector. The MBP-PIF3 construct was reported previously ${ }^{47}$.

To generate HY5-nLUC constructs, full-length and truncated HY5 fragments were cloned into the KpnI/SalI sites of the pCAMBIA1300- $n L U C$ vector. The cLUC-BIN2, cLUC-BIL1, and $c L U C-B I L 2$ constructs were reported previously 48

To generate the EXP2 $2_{\text {pro }}-L U C$ construct, a DNA fragment with $1021 \mathrm{bp}$ upstream of the ATG together with 49 bp downstream of the ATG of EXP2 was amplified and cloned into the KpnI/NcoI sites of the pGreenII 0800-LUC vector ${ }^{49}$.

The bzrlbes 1 double mutant was generated by egg cell-specific promotercontrolled CRISPR/Cas $9^{31}$, and then crossed with hy5 to get a hy5bzrlbes 1 triple mutant. To generate transgenic plants overexpressing $3 \times \mathrm{HA}$-tagged HY5, the promoter of Arabidopsis UBQ10 was amplified from genomic DNA ${ }^{50}$, then the

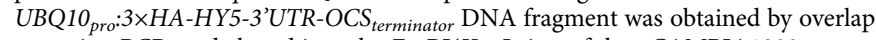
extension PCR, and cloned into the EcoRI/KpnI sites of the pCAMBIA1300 vector. The UBQ10 pro $: 3 \times H A-H Y 5^{L 137 D}$ fragment was amplified by primer-based sitedirected mutagenesis and then also cloned into the EcoRI/KpnI sites of the pCAMBIA1300 vector. For stable transformation, Agrobacterium tumefaciens strain GV3101 carrying the construct was then used to transform the transgenes into Col, Ws, and other related mutants using the floral dip method ${ }^{51}$.

Homozygous lines were screened based on hygromycin resistance. Generation of BIN2-FLAG transgenic plants was reported previously ${ }^{48}$. All the cloning and genotyping primers are listed in Supplementary Table 1.

Western blot and antibodies. For protein extraction, seedlings were frozen in liquid nitrogen, ground into powder, then resuspended in $2 \times$ SDS buffer $(0.125 \mathrm{M}$ Tris- $\mathrm{HCl}$ [pH 6.8], 4\% SDS, $20 \%$ glycerol, $1 \times$ cocktail of protease and phosphatase inhibitors, $1 \mathrm{mM}$ PMSF). Samples were heated for $10 \mathrm{~min}$ at $65^{\circ} \mathrm{C}$, then centrifuged at $13,000 \times g$ for $10 \mathrm{~min}$ at room temperature. The supernatants were transferred into new tubes, and the total protein concentrations were determined by the BCA method. Equal amounts of total proteins were separated in $10 \%$ sodium dodecyl sulfate (SDS)-polyacrylamide gels and then transferred onto PVDF membranes. The subsequent immunoblots were performed as previously described ${ }^{52}$. 
a

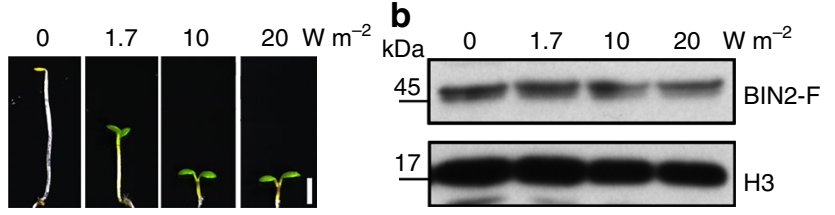

C
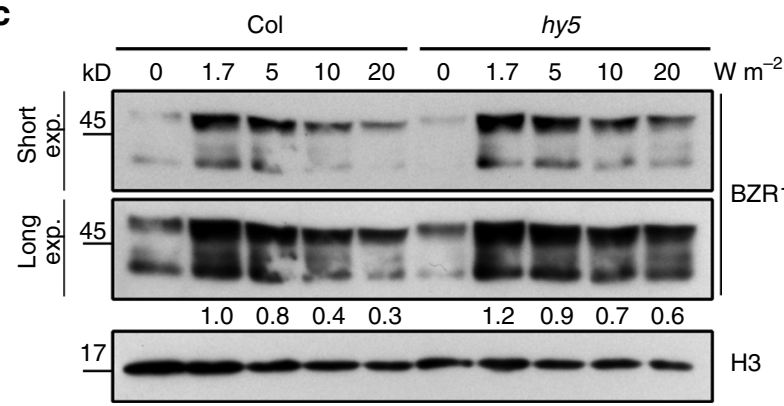

d
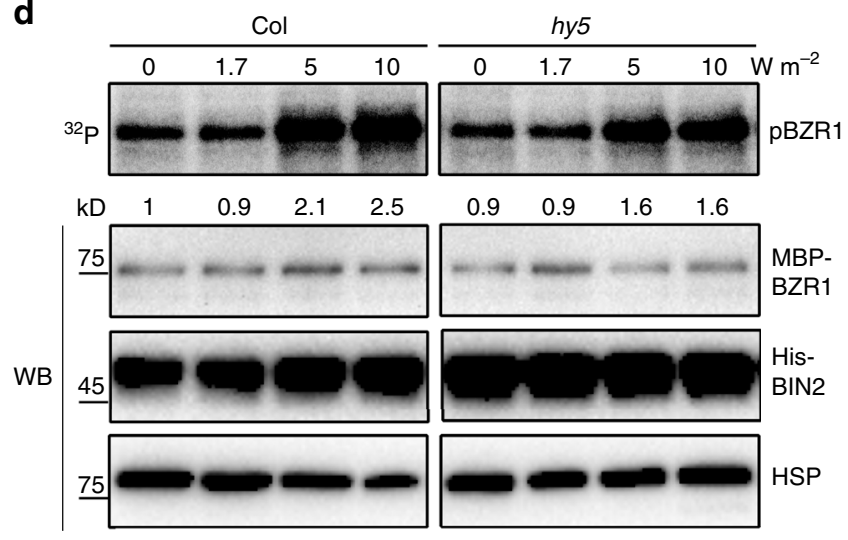

MBPBZR1

e

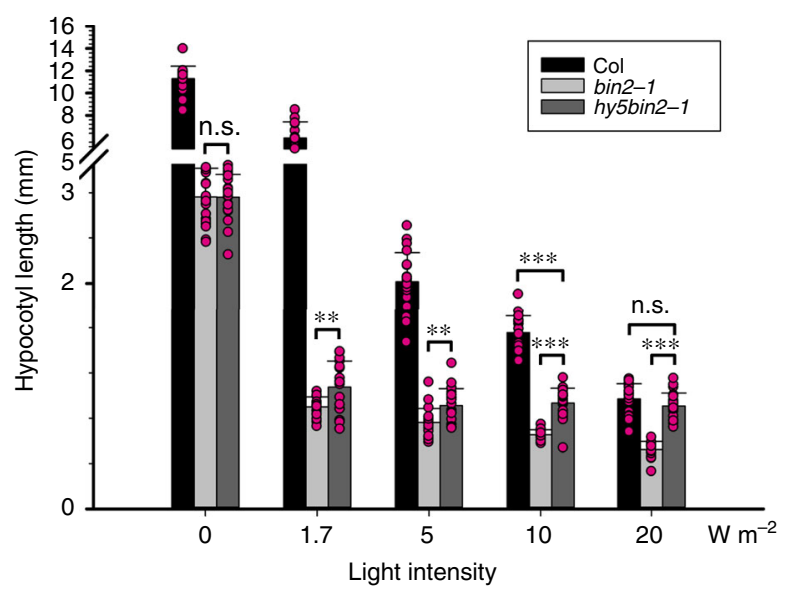

Antibodies used in this study were anti-HY5 (A gift from Rongcheng Lin's lab, 1:1000 dilution), anti-BZR1 (A gift from Jianming Li's lab, 1:1000 dilution), antiHistone H3 (05-499, Millipore, 1:1000 dilution), anti-HSP (AbM51099-31-PU, Beijing Protein Innovation, 1:5000 dilution), anti-HA (H9658-.2 ML, SigmaAldrich, 1:2000 dilution), anti-Flag (F3165-.2MG, Sigma-Aldrich, 1:2000 dilution), Anti-phospho-GSK3 (Tyr279/Tyr216) (05-413, Millipore, 1:1000 dilution), antiMBP (\#E8031S, New England Biolabs, 1:5000 dilution), anti-His (H1029-.2ML, Sigma-Aldrich, 1:2000 dilution), and anti-GST (\#2625, Cell Signaling Technology, $1: 1000$ dilution)

Yeast one-hybrid and two-hybrid assays. Yeast one-hybrid assays were performed as described previously ${ }^{11}$. Briefly, pB42AD-HY5 (effector) and pLacZi$E X P 2_{\text {pro }}$ (reporter) were co-transformed into yeast strain EGY48, the transformants were plated on minimal synthetic defined (SD) base supplemented with the -Ura/ -Trp dropout (DO) mix and X-gal (5-bromo-4-chloro-3-indolyl- $\beta$-D-galactopyranoside) for blue color development. Yeast two-hybrid assays based on the LexA
Fig. 7 The HY5-BIN2 module regulates hypocotyl elongation subtly in varying light intensities. a Morphology of Col grown under different light intensities for 6 days. Scale bar, $1 \mathrm{~mm}$. b BIN2-FLAG (BIN2-F) protein levels in transgenic plants that overexpress BIN2. Transgenic seedlings were grown for 6 days under the indicated light intensities. H3 was used as a loading control. c BZR1 protein levels in Col and hy5. Seedlings were grown for 6 days under the indicated light intensities. H3 was used as a loading control. Numbers under lanes indicate relative BZR1 band intensities that were quantified for each panel. d Cell-free kinase assay showing BIN2mediated phosphorylation of BZR1 in the total lysates of Col and hy5, which were grown under the indicated light intensities. Numbers under lanes indicated the relative levels of BIN2-mediated phosphorylation of BZR1. HSP was used as a loading control. e Hypocotyl lengths of mutants. Seedlings were grown for 6 days under the indicated light intensities. Error bars represent SD, $n \geq 20$. Asterisks indicate the $P$ value of two-tailed Student's $t$-test; ${ }^{\star \star} P<0.01 ;{ }^{\star \star \star} P<0.001$; n.s., not significant. Experiments were performed three times with similar results. Source data are provided as a source data file.

system were performed according to the standard protocol (Clontech). The yeast strain used in this study was EGY48 containing p8oplacZ vector.

In vitro pull-down assays. For in vitro pull-down assays, $1 \mu \mathrm{g}$ GST-HY5 and $1 \mu \mathrm{g}$ His-BIN2 proteins were incubated in $1 \mathrm{ml}$ binding buffer $(25 \mathrm{mM}$ Tris- $\mathrm{Cl}$ [pH 7.5], $100 \mathrm{mM} \mathrm{NaCl}, 0.1 \% \mathrm{NP} 40$ ) at $4{ }^{\circ} \mathrm{C}$ for $1 \mathrm{~h}$. Ten microliters GST beads pre-washed with PBS were added into the binding buffer and incubated with the proteins for another $1 \mathrm{~h}$. After that, the beads were washed three times with washing buffer ( $25 \mathrm{mM}$ Tris-Cl [pH 7.5], $500 \mathrm{mM} \mathrm{NaCl}$ ) at $4{ }^{\circ} \mathrm{C}$. GST beads were heated for $5 \mathrm{~min}$ in $1 \times$ SDS loading buffer at $100^{\circ} \mathrm{C}$. The eluted proteins were then analyzed by immuno-blotting using anti-GST and anti-His antibodies.

Co-IP assays. For in vivo Co-IP assays, $0.5 \mathrm{~g}$ seedlings were frozen in liquid nitrogen, ground into powder, and added into lysis buffer $(25 \mathrm{mM}$ Tris- $\mathrm{HCl}[\mathrm{pH}$ 7.5], $150 \mathrm{mM} \mathrm{NaCl}, 1 \mathrm{mM}$ EDTA, 10\% glycerol, $0.5 \%$ Tween-20). Samples were centrifuged at $15,000 \times g$ for $10 \mathrm{~min}$, and then total protein concentrations of the supernatants were determined by the Bradford method. One milligram total proteins were incubated with $10 \mu \mathrm{l}$ anti-FLAG beads in $1 \mathrm{ml}$ lysis buffer for $3 \mathrm{~h}$ at $4^{\circ} \mathrm{C}$ After that, the beads were washed three times with washing buffer $(25 \mathrm{mM}$ Tris-Cl [pH 7.5], $150 \mathrm{mM} \mathrm{NaCl}, 0.1 \%$ Tween-20) at $4{ }^{\circ} \mathrm{C}$. The proteins were eluted from the beads with the addition of $3 \times$ FLAG peptide (F4799, Sigma-Aldrich). Eluted proteins were analyzed by immuno-blotting using anti-BZR1, anti-FLAG. and antiHSP antibodies.

For semi-in vivo Co-IP assays, total proteins of Col or BIN2-FLAG seedlings were extracted using the lysis buffer $(25 \mathrm{mM}$ Tris- $\mathrm{HCl}[\mathrm{pH}$ 7.5], $150 \mathrm{mM} \mathrm{NaCl}$, $1 \mathrm{mM}$ EDTA, $10 \%$ glycerol, $0.1 \%$ NP40). Five hundred micrograms total proteins were incubated with $8 \mu \mathrm{g}$ GST-HY5 protein in $0.5 \mathrm{ml}$ lysis buffer for $2 \mathrm{~h}$ at $4{ }^{\circ} \mathrm{C}$, then $10 \mu \mathrm{l}$ anti-FLAG beads were added and incubated with the mixture for three more hours at $4{ }^{\circ} \mathrm{C}$. After that, the beads were washed three times with 25 column volumes of washing buffer ( $25 \mathrm{mM}$ Tris- $\mathrm{Cl}$ [pH 7.5], $500 \mathrm{mM} \mathrm{NaCl}, 1 \mathrm{mM}$ EDTA, $10 \%$ glycerol). The proteins were eluted with $3 \times$ FLAG peptide and analyzed by immuno-blotting using anti-GST, anti-FLAG, and anti-HSP antibodies.

LCI assay. The LCI assays were performed as previously described with some modifications ${ }^{53}$. In brief, GV3101 colonies containing different constructs were inoculated into $5 \mathrm{ml} \mathrm{LB}$ medium supplemented with kanamycin, and grown at $28^{\circ} \mathrm{C}$ for $16 \mathrm{~h}$. In all, $0.1 \mathrm{ml}$ of the cultures were transferred to $5 \mathrm{ml} \mathrm{LB}$ supplemented with $10 \mathrm{mM}$ MES (pH 5.6) and $40 \mu \mathrm{M}$ acetosyringone. Bacteria were grown at $28^{\circ} \mathrm{C}$ for $16 \mathrm{~h}$, and harvested by centrifugation. The bacteria were resuspended in buffer containing $10 \mathrm{mM}$ MES (pH 5.6), $10 \mathrm{mM} \mathrm{MgCl}_{2}$, and $100 \mu \mathrm{M}$ acetosyringone. Related bacteria were mixed and each bacterium had a final concentration of $\mathrm{OD}_{600}=0.5$. The bacteria were kept at room temperature for $3-5 \mathrm{~h}$ without shaking. Infiltration was performed with a 2 -ml syringe without needle. The plants were subsequently kept away from light for $12 \mathrm{~h}$, and then grown in the light for 2 days before the analysis. The luciferase signals were analyzed with Night SHADE LB 985 (Berthold Technologies).

Protoplast transient expression assays. Protoplasts from Arabidopsis mesophyll cells were prepared and transformed as described previously ${ }^{54}$. The effector plasmid UBQ10 pro:HA-HY5, reporter EXP2 ${ }_{p r o}: L U C$, and control 35S:REN were cotransformed into protoplasts and incubated under weak light for $12 \mathrm{~h}$. Then the protoplasts were harvested, and the luminescent signals of LUC and REN were 
detected with the Dual-Glo ${ }^{\circledast}$ Luciferase Assay System (E2920, Promega). The LUC expression levels were quantified as the ratio of LUC/REN enzyme activities.

Quantitative real-time PCR. Total RNA was extracted from seedlings using RNeasy Plant Mini Kits (74904, QIAGEN). One microgram of total RNA was incubated with DNase I at $37^{\circ} \mathrm{C}$ to remove the genomic DNA, and then the prepared RNA was used as a template for RevertAid Reverse Transcriptase (EP0442, Thermo Scientific) to generate first-strand cDNA. qPCR was performed using a 7500 Fast Real-Time PCR System (Applied Biosystems). The CT values were used to calculate the expression levels of different genes normalized to PP2A. All the RT-qPCR primers are listed in Supplementary Table 1.

In vitro kinase assays. In vitro and cell-free kinase assays were performed as previously described with some modification $s^{55}$. For in vitro kinase assays, $50 \mathrm{ng}$ His-BIN2 were pre-mixed with HY5-His, the mole ratio of BIN2/HY5 ranging from 1:1 to 1:8 as indicated, then $1 \mu \mathrm{g}$ MBP-BZR1 was incubated with the mixture in $20 \mu$ linase buffer $\left(20 \mathrm{mM}\right.$ Tris- $\mathrm{HCl}$ [pH 7.5], $100 \mathrm{mM} \mathrm{NaCl}, 12 \mathrm{mM} \mathrm{MgCl}_{2}$, $0.1 \mathrm{mM}$ ATP, $\left.0.2 \mu \mathrm{Ci}\left[\gamma^{-32} \mathrm{P}\right] \mathrm{ATP}\right)$ at $30^{\circ} \mathrm{C}$ for $30 \mathrm{~min}$. For the cell-free kinase assays, total proteins were extracted from $\mathrm{Col}$ and hy5, respectively, in the buffer (20 mM Tris- $\mathrm{HCl}$ [pH 7.5], $100 \mathrm{mM} \mathrm{NaCl}, 12 \mathrm{mM} \mathrm{MgCl}$ ) containing $1 \times$ cocktail of protease and phosphatase inhibitors, and $1 \mathrm{mM}$ PMSF. Five micrograms total proteins were incubated with $1 \mu \mathrm{g}$ MBP-BZR1 and $50 \mathrm{ng}$ His-BIN2 in $20 \mu \mathrm{l}$ kinase buffer containing $1 \times$ cocktail of protease and phosphatase inhibitors. After incubation at $30^{\circ} \mathrm{C}$ for $30 \mathrm{~min}$, the reactions were terminated in $1 \times$ SDS loading buffer, and samples were separated in $10 \%$ SDS-polyacrylamide gels. ${ }^{32} \mathrm{P}$ signals were detected with Typhoon FLA7000 (GE Healthcare).

Computational methods. The monomeric HY5 and BIN2 proteins were modeled using JACKAL ${ }^{56}$ and ITASSER programs ${ }^{57}$. The models with the highest scores were chosen as the results. The predicted structure was then minimized using molecular dynamics simulation package Gromacs $4.5^{58}$

The complex formed by the interaction between HY5 and BIN2 was predicted by the docking method HoDock ${ }^{59}$, which incorporates an initial rigid docking and a refined semi-flexible docking. A total of 200,000 complex structures were generated and scored to select the final correct complex structure model. The docked complex model was also minimized using the molecular dynamics simulation package Gromacs 4.5. The interface contact residues were calculated by the Cartesian coordinates of non-hydrogen heavy atoms $\mathrm{C}, \mathrm{N}$, and $\mathrm{O}$. Those residues with at least one pair of heavy atoms within $4 \AA$ were denoted as interface contact residues.

The minimized monomeric BIN2 and complex BIN2-HY5 structures were used to simulate the functional motions by a coarse-grained anisotropic network model $^{60}$. All data were depicted and presented using PyMOL software. The movies were created using the PyMOL frame pictures and Matlab script.

Primer sequences. The sequences for all primers used in this study are listed in Supplementary Table 1 .

Reporting summary. Further information on research design is available in the Nature Research Reporting Summary linked to this article.

\section{Data availability}

The source data generated and analyzed in this study are provided as a Source Data file. All relevant data are available from the corresponding authors upon request. There are no restrictions on data availability.

Received: 13 August 2019; Accepted: 4 March 2020;

Published online: 27 March 2020

\section{References}

1. McNellis, T. W. \& Deng, X.-W. Light control of seedling morphogenetic pattern. Plant Cell 7, 1749-1761 (1995).

2. Hersch, M. et al. Light intensity modulates the regulatory network of the shade avoidance response in Arabidopsis. Proc. Natl Acad. Sci. USA 111, 6515-6520 (2014).

3. Osterlund, M. T., Hardtke, C. S., Wei, N. \& Deng, X. W. Targeted destabilization of HY5 during light-regulated development of Arabidopsis. Nature 405, 462-466 (2000).

4. Chattopadhyay, S., Ang, L.-H., Puente, P., Deng, X.-W. \& Wei, N. Arabidopsis bZIP protein HY5 directly interacts with light-responsive promoters in mediating light control of gene expression. Plant Cell 10, 673-683 (1998).

5. Oyama, T., Shimura, Y. \& Okada, K. The Arabidopsis HY5 gene encodes a bZIP protein that regulates stimulus-induced development of root and hypocotyl. Gene Dev. 11, 2983-2995 (1997).
6. Jakoby, M. et al. bZIP transcription factors in Arabidopsis. Trends Plant Sci. 7, 106-111 (2002).

7. Yoon, M.-K., Kim, H.-M., Choi, G., Lee, J.-O. \& Choi, B.-S. Structural basis for the conformational integrity of the Arabidopsis thaliana HY5 leucine zipper homodimer. J. Biol. Chem. 282, 12989-13002 (2007).

8. Lee, J. et al. Analysis of transcription factor HY5 genomic binding sites revealed its hierarchical role in light regulation of development. Plant Cell 19, 731-749 (2007).

9. Singh, A., Ram, H., Abbas, N. \& Chattopadhyay, S. Molecular interactions of GBF1 with HY5 and HYH proteins during light-mediated seedling development in Arabidopsis thaliana. J. Biol. Chem. 287, 25995-26009 (2012).

10. Holm, M., Ma, L.-G., Qu, L.-J. \& Deng, X.-W. Two interacting bZIP proteins are direct targets of COP1-mediated control of light-dependent gene expression in Arabidopsis. Gene Dev. 16, 1247-1259 (2002).

11. Lin, F. et al. B-BOX DOMAIN PROTEIN28 negatively regulates photomorphogenesis by repressing the activity of transcription factor HY5 and undergoes COP1-mediated degradation. Plant Cell 30, 2006-2019 (2018).

12. Hardtke, C. S. et al. HY5 stability and activity in Arabidopsis is regulated by phosphorylation in its COP1 binding domain. EMBO J. 19, 4997-5006 (2000).

13. Abbas, N., Maurya, J. P., Senapati, D., Gangappa, S. N. \& Chattopadhyay, S. Arabidopsis CAM7 and HY5 physically interact and directly bind to the HY5 promoter to regulate its expression and thereby promote photomorphogenesis. Plant Cell 26, 1036-1052 (2014).

14. Yang, Y. et al. UVR8 interacts with WRKY36 to regulate HY5 transcription and hypocotyl elongation in Arabidopsis. Nat. Plants 4, 98-107 (2018).

15. Yuan, T. T., Xu, H. H., Zhang, Q., Zhang, L. Y. \& Lu, Y. T. The COP1 target SHI-RELATED SEQUENCE5 directly activates photomorphogenesispromoting genes. Plant Cell 30, 2368-2382 (2018).

16. Li, J., Nagpal, P., Vitart, V., McMorris, T. C. \& Chory, J. A role for brassinosteroids in light-dependent development of Arabidopsis. Science 272, 398-401 (1996).

17. Li, J., Nam, K. H., Vafeados, D. \& Chory, J. BIN2, a new brassinosteroidinsensitive locus in Arabidopsis. Plant Physiol. 127, 14-22 (2001).

18. Wang, Z.-Y. et al. Nuclear-localized BZR1 mediates brassinosteroid-induced growth and feedback suppression of brassinosteroid biosynthesis. Dev. Cell 2, 505-513 (2002).

19. Vert, G. \& Chory, J. Downstream nuclear events in brassinosteroid signalling Nature 441, 96-100 (2006).

20. He, J.-X., Gendron, J. M., Yang, Y., Li, J. \& Wang, Z.-Y. The GSK3-like kinase BIN2 phosphorylates and destabilizes BZR1, a positive regulator of the brassinosteroid signaling pathway in Arabidopsis. Proc. Natl Acad. Sci. USA 99, 10185-10190 (2002)

21. Kim, B. et al. Darkness and gulliver2/phyB mutation decrease the abundance of phosphorylated BZR1 to activate brassinosteroid signaling in Arabidopsis. Plant J. 77, 737-747 (2014).

22. Oh, E., Zhu, J.-Y. \& Wang, Z.-Y. Interaction between BZR1 and PIF4 integrates brassinosteroid and environmental responses. Nat. Cell Biol. 14, 802-809 (2012).

23. Luo, X.-M. et al. Integration of light- and brassinosteroid-signaling pathways by a GATA transcription factor in Arabidopsis. Dev. Cell 19, 872-883 (2010).

24. Liang, T. et al. UVR8 interacts with BES1 and BIM1 to regulate transcription and photomorphogenesis in Arabidopsis. Dev. Cell 44, 512-523 e515 (2018).

25. Wang, W. et al. Photoexcited CRYPTOCHROME 1 interacts with dephosphorylated BES1 to regulate brassinosteroid signaling and photomorphogenesis in Arabidopsis. Plant Cell 30, 1989-2005 (2018).

26. He, G., Liu, J., Dong, H. \& Sun, J. The blue light receptor CRY1 interacts with BZR1 and BIN2 to modulate the phosphorylation and nuclear function of BZR1 in repressing BR signaling in Arabidopsis. Mol. Plant 12, 689-703 (2019).

27. Li, Q.-F. \& He, J.-X. BZR1 interacts with HY5 to mediate brassinosteroid- and light-regulated cotyledon opening in Arabidopsis in darkness. Mol. Plant $\mathbf{9}$, 113-125 (2015).

28. Peng, P., Yan, Z., Zhu, Y. \& Li, J. Regulation of the Arabidopsis GSK3-like kinase BRASSINOSTEROID-INSENSITIVE 2 through proteasome-mediated protein degradation. Mol. Plant 1, 338-346 (2008).

29. De Rybel, B. et al. Chemical inhibition of a subset of Arabidopsis thaliana GSK3-like kinases activates brassinosteroid signaling. Chem. Biol. 16, 594-604 (2009).

30. Kim, T.-W. \& Wang, Z.-Y. Brassinosteroid signal transduction from receptor kinases to transcription factors. Annu. Rev. Plant Biol. 61, 681-704 (2010).

31. Wang, J. \& Chen, H. A novel CRISPR/Cas9 system for efficiently generating Cas9-free multiplex mutants in Arabidopsis. aBIOTECH 1, 6-14 (2020).

32. Xu, X. M. et al. Convergence of light and chloroplast signals for de-etiolation through ABI4-HY5 and COP1. Nat. Plants 2, 7 (2016).

33. Kim, T.-W. et al. Brassinosteroid signal transduction from cell-surface receptor kinases to nuclear transcription factors. Nat. Cell Biol. 11, 1254-1260 (2009). 
34. Li, J. et al. Arabidopsis transcription factor ELONGATED HYPOCOTYL5 plays a role in the feedback regulation of phytochrome A signaling. Plant Cell 22, 3634-3649 (2010).

35. Jing, Y. J. et al. Arabidopsis chromatin remodeling factor PICKLE interacts with transcription factor HY5 to regulate hypocotyl cell elongation. Plant Cell 25, 242-256 (2013).

36. Wei, Z. et al. Brassinosteroid biosynthesis is modulated via a transcription factor cascade of COG1, PIF4, and PIF5. Plant Physiol. 174, 1260-1273 (2017).

37. Martínez, C. et al. PIF4-induced BR synthesis is critical to diurnal and thermomorphogenic growth. EMBO J. 37, e99552 (2018).

38. Hao, Y. H., Wang, H. J., Qiao, S. L., Leng, L. N. \& Wang, X. L. Histone deacetylase HDA6 enhances brassinosteroid signaling by inhibiting the BIN2 kinase. Proc. Natl Acad. Sci. USA 113, 10418-10423 (2016).

39. Anne, P. et al. OCTOPUS negatively regulates BIN2 to control phloem differentiation in Arabidopsis thaliana. Curr. Biol. 25, 2584-2590 (2015).

40. Zhu, J. Y. et al. The F-box protein KIB1 mediates brassinosteroid-induced inactivation and degradation of GSK3-like kinases in Arabidopsis. Mol. Cell 66, 648-657 (2017).

41. Saidi, Y., Hearn, T. J. \& Coates, J. C. Function and evolution of 'green' GSK3/ Shaggy-like kinases. Trends Plant Sci. 17, 39-46 (2012).

42. Hughes, K., Nikolakaki, E., Plyte, S. E., Totty, N. F. \& Woodgett, J. R. Modulation of the glycogen synthase kinase- 3 family by tyrosine phosphorylation. EMBO J. 12, 803-808 (1993).

43. Cole, A., Frame, S. \& Cohen, P. Further evidence that the tyrosine phosphorylation of glycogen synthase kinase-3 (GSK3) in mammalian cells is an autophosphorylation event. Biochem. J. 377, 249-255 (2004).

44. Lochhead, P. A. et al. A chaperone-dependent GSK3 beta transitional intermediate mediates activation-loop autophosphorylation. Mol. Cell 24, 627-633 (2006).

45. Chen, H. et al. Integration of light and abscisic acid signaling during seed germination and early seedling development. Proc. Natl Acad. Sci. USA 105, 4495-4500 (2008).

46. Yan, Z., Zhao, J., Peng, P., Chihara, R. K. \& Li, J. BIN2 functions redundantly with other Arabidopsis GSK3-like kinases to regulate brassinosteroid signaling. Plant Physiol. 150, 710-721 (2009).

47. Lin, R. et al. Transposase-derived transcription factors regulate light signaling in Arabidopsis. Science 318, 1302-1305 (2007).

48. Ling, J.-J., Li, J., Zhu, D. \& Deng, X. W. Noncanonical role of Arabidopsis COP1/SPA complex in repressing BIN2-mediated PIF3 phosphorylation and degradation in darkness. Proc. Natl Acad. Sci. USA 114, 3539-3544 (2017).

49. Hellens, R. P. et al. Transient expression vectors for functional genomics, quantification of promoter activity and RNA silencing in plants. Plant Methods 1, 13 (2005).

50. Grefen, C. et al. A ubiquitin-10 promoter-based vector set for fluorescent protein tagging facilitates temporal stability and native protein distribution in transient and stable expression studies. Plant J. 64, 355-365 (2010).

51. Clough, S. J. \& Bent, A. F. Floral dip: a simplified method for Agrobacterium-mediated transformation of Arabidopsis thaliana. Plant J. 16, 735-743 (1998).

52. Zhu, D. et al. Biochemical characterization of Arabidopsis complexes containing CONSTITUTIVELY PHOTOMORPHOGENIC1 and SUPPRESSOR OF PHYA proteins in light control of plant development. Plant Cell 20, 2307-2323 (2008).

53. Chen, H. M. et al. Firefly luciferase complementation imaging assay for protein-protein interactions in plants. Plant Physiol. 146, 368-376 (2008)

54. Yoo, S.-D., Cho, Y.-H. \& Sheen, J. Arabidopsis mesophyll protoplasts: a versatile cell system for transient gene expression analysis. Nat. Protoc. 2, 1565-1572 (2007).

55. Hu, Y. R. \& Yu, D. Q. BRASSINOSTEROID INSENSITIVE2 interacts with ABSCISIC ACID INSENSITIVE5 to mediate the antagonism of brassinosteroids to abscisic acid during seed germination in Arabidopsis. Plant Cell 26, 4394-4408 (2014).
56. Xiang, Z., Soto, C. S. \& Honig, B. Evaluating conformational free energies: the colony energy and its application to the problem of loop prediction. Proc. Natl Acad. Sci. USA 99, 7432-7437 (2002).

57. Yang, J. et al. The I-TASSER Suite: protein structure and function prediction. Nat. Methods 12, 7-8 (2015).

58. Pronk, S. et al. GROMACS 4.5: a high-throughput and highly parallel open source molecular simulation toolkit. Bioinformatics 29, 845-854 (2013).

59. Gong, X. et al. Protein-protein docking with binding site patch prediction and network-based terms enhanced combinatorial scoring. Proteins 78, 3150-3155 (2010).

60. Eyal, E., Yang, L.-W. \& Bahar, I. Anisotropic network model: systematic evaluation and a new web interface. Bioinformatics 22, 2619-2627 (2006).

\section{Acknowledgements}

We are grateful to Jianming Li (University of Michigan) for providing the BZR1 antibody and the bin2-1 and bin2-3bil1bil2 mutants. We thank Dr. Xiaodong Su, Dr. Junyu Xiao, and Dr. Ningning Li for their helpful discussions and comments and Shitang Huang for help with experiments involving $\left[\gamma_{-}{ }^{32} \mathrm{P}\right]$ ATP. This work was supported by the National Key R \& D Program of China Grants 2017YFA0503800 and 2016YFA0500800; the National Natural Science Foundation of China Grants 31621001, 31330048, and 31670725, and in part by the Postdoctoral Fellowship of Peking-Tsinghua Center for Life Sciences.

\section{Author contributions}

J.L., D.Z., and X.W.D. designed research; J.L., Y.G., C.L., and N.Q. performed experiments; X.G. carried out the computer modeling; J.L., Y.F., J.-J.L., D.Z., and X.W.D analyzed data; J.L., W.T., X.G., D.Z., and X.W.D. wrote the paper.

\section{Competing interests}

The authors declare no competing interests.

\section{Additional information}

Supplementary information is available for this paper at https://doi.org/10.1038/s41467 020-15394-7.

Correspondence and requests for materials should be addressed to X.G., D.Z. or X.W.D.

Peer review information Nature Communications thanks the anonymous reviewers for their contribution to the peer review of this work.

Reprints and permission information is available at http://www.nature.com/reprints

Publisher's note Springer Nature remains neutral with regard to jurisdictional claims in published maps and institutional affiliations.

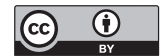

Open Access This article is licensed under a Creative Commons Attribution 4.0 International License, which permits use, sharing, adaptation, distribution and reproduction in any medium or format, as long as you give appropriate credit to the original author(s) and the source, provide a link to the Creative Commons license, and indicate if changes were made. The images or other third party material in this article are included in the article's Creative Commons license, unless indicated otherwise in a credit line to the material. If material is not included in the article's Creative Commons license and your intended use is not permitted by statutory regulation or exceeds the permitted use, you will need to obtain permission directly from the copyright holder. To view a copy of this license, visit http://creativecommons.org/ licenses/by/4.0/.

(c) The Author(s) 2020 